\title{
Analysis of adsorption of a carbon steel corrosion inhibitor in model stratum waters by means of a polylogarithmic isotherm
}

\author{
L.E. Tsygankova, ${ }^{1}{ }^{(1) *}$ N. Alshikha, ${ }^{1}$ M. Vigdorowitsch ${ }^{2}$ and I.V. Zarapina ${ }^{3}$ \\ ${ }^{1}$ Derzhavin State University, Internatsyonalnaya str., 33, 392000 Tambov, Russian \\ Federation \\ ${ }^{2}$ Angara GmbH, In der Steele 2, D-40599, Düsseldorf, Germany \\ ${ }^{3}$ Tambov State Technical University, Sovetskaya str., 106, 392000 Tambov, Russian \\ Federation \\ *E-mail: vits21@mail.ru
}

\begin{abstract}
Using the methods of gravimetry, potentiodynamic polarization, and impedance spectroscopy, the protective ability of inhibitory composition INCORGAZ-111 with respect to hydrogen sulfide corrosion of carbon steel St3 in NACE and Samotlor oil field (M1) model stratum waters is studied. The concentration of hydrogen sulfide was 50-400 mg/L. The inhibitory composition INCORGAZ-111 is a condensation product of tall oil fatty acids and polyamines in the salt form in a water-alcohol solvent (Developed by "INCORGAZ" LLC). The INCORGAZ-111 inhibitor exhibits a relatively high protective effect in NACE and M1 media reaching 80-90\% at a concentration of $200 \mathrm{mg} / \mathrm{L}$. Analysis of the polarization curves in the solutions studied indicates that the INCORGAZ-111 inhibitor slows down the anodic process in both media. According to impedance data, the charge transfer resistance in the anodic reaction increases with increasing concentration of INCORGAZ-111 inhibitor. The surface coverage was determined on the basis of the electric double layer capacitance in the presence of the inhibitor. Its adsorption was interpreted using the polylogarithmic isotherm with a big energy gradient of adsorption centers, that indicates a linear energy heterogeneity of the surface. The constants of adsorption equilibrium, energy gradient and energy dispersion of adsorption centers as well as adsorption free energy were calculated.
\end{abstract}

Keywords: corrosion, stratum water, hydrogen sulfide, inhibitor, capacitance, adsorption, surface coverage, polylogarithmic isotherm.

Received: October 16, 2020. Published: February 2, 2021

doi: $\underline{10.17675 / 2305-6894-2021-10-1-7}$

\section{Introduction}

The corrosiveness of stratum water in oil production wells is often due to the presence of hydrogen sulfide. One of the most economical methods of anticorrosive protection of metal equipment is the use of inhibitors. New inhibitor compositions are constantly being developed, focused on the use of domestic raw materials and multifunctional action. A variety of methods are used to study their effectiveness. 
The most commonly used methods are gravimetric corrosion tests and electrochemical polarization measurements. As imitations of stratum waters of oil and gas fields, the following model media are often used: NACE, recommended by the National Association of Corrosion Engineers of the United States, and the synthetic stratum water of the Samotlor Oil Field (M1). They differ in salt composition and magnitude of the $\mathrm{pH}$ index. For the first one, $\mathrm{pH}=3.6$, and for the second it is 6 . This difference in $\mathrm{pH}$ can significantly change the nature and concentration of the protonated form of hydrogen sulfide corrosion inhibitors, which are often nitrogen-containing compounds.

In addition, the kinetics of iron ionization depends on $\mathrm{pH}$ of the solutions. The concentration of chloride ions in these media is also very different, and these are surfaceactive ions, which can significantly affect the kinetics of metal ionization. The features of these environments are discussed in [1].

The use of electrochemical impedance spectroscopy makes it possible to confirm adsorption of the inhibitor on protected metal and to propose the corresponding adsorption isotherm.

The dependence of the metal surface coverage with an inhibitor on the logarithm of its concentration is often approximated by a straight line. This type of dependence is characteristic of the Temkin isotherm. View of the Temkin isotherm for the region of average coverages:

$$
B C=\exp (f \Theta),
$$

where $f$ is the factor of the energy inhomogeneity of the surface, which characterizes the change in the heat of adsorption with the filling of the surface, $B$ is the constant of adsorption equilibrium. For the interpretation of experimental data, both the Temkin isotherm $[2,3]$ and the Frumkin isotherm [4] are often used:

$$
B C=[\Theta /(1-\Theta)] \exp (-2 a \Theta),
$$

where $a$ is the attraction constant characterizing the interaction between adsorbed particles.

In this paper, we consider the inhibitory ability of a product manufactured by "INCORGAZ" LLC (St. Petersburg).

The aim of the study is to elucidate the nature of adsorption of inhibitory composition INCORGAZ-111, which is the condensation product of tall oil fatty acids and polyamines, on carbon steel in simulated NACE and M1 stratum waters with hydrogen sulfide additives.

\section{Experimental methods}

The research was conducted on samples of steel St3 with composition, mass.\%: $\mathrm{Fe}-98.36$; $\mathrm{C}-0.2 ; \mathrm{Mn}-0.5 ; \mathrm{Si}-0.15 ; \mathrm{P}-0.04 ; \mathrm{S}-0.05 ; \mathrm{Cr}-0.3 ; \mathrm{Ni}-0.2 ; \mathrm{Cu}-0.2$.

The following media were studied as the working solutions: NACE composition, $5 \mathrm{~g} / \mathrm{L}$ $\mathrm{NaCl} ; 0.25 \mathrm{~g} / \mathrm{L} \mathrm{CH}_{3} \mathrm{COOH}$, and model water M1 (simulation of stratum water of Samotlor oil field) with the content, $\mathrm{g} / \mathrm{L}: \mathrm{NaCl} 17 ; \mathrm{CaCl}_{2} 0.2 ; \mathrm{MgCl}_{2} \cdot 6 \mathrm{H}_{2} \mathrm{O} 0.2 ; \mathrm{NaHCO}_{3} 0.8$. Various amounts of $\mathrm{H}_{2} \mathrm{~S}(50-400 \mathrm{mg} / \mathrm{L})$ were added to the media investigated. Distilled water was 
used, hydrochloric acid and salts were of "chemically pure" qualifications. Hydrogen sulfide was produced directly in the working solution, for which calculated amounts of $\mathrm{Na}_{2} \mathrm{~S}$ and $\mathrm{HCl}$ were introduced, corresponding to the equation:

$$
\mathrm{Na}_{2} \mathrm{~S}+2 \mathrm{HCl}=\mathrm{H}_{2} \mathrm{~S}+2 \mathrm{NaCl}
$$

The concentration of hydrogen sulfide was controlled iodometrically. The method of gravimetric corrosion testing is generally accepted based on the calculation of the corrosion rate from the weight loss of the samples during the test.

After exposure, the electrodes were etched with a $15 \% \mathrm{HCl}$ solution containing $1 \mathrm{~g} / \mathrm{L}$ of urotropine and $1 \mathrm{~g} / \mathrm{L}$ of $\mathrm{KI}$, then they were washed with water, dried with filter paper and treated with a soft eraser.

The duration of the experiments was 1 day. The protective effect of inhibitor $(Z)$ was calculated by the formula:

$$
\left.Z, \%=\left[\left(K_{0}-K_{\mathrm{i}}\right) / K_{0}\right)\right] \cdot 100 \%,
$$

where $K_{0}$ and $K_{\mathrm{i}}$ are the corrosion rates in the absence and in the presence of inhibitor in the test solutions, respectively.

Potentiodynamic measurements at a potential sweep rate of $0.66 \mathrm{mV} / \mathrm{s}$ were carried out in a three-electrode electrochemical cell made of Pyrex glass with a platinum auxiliary electrode and silver-silver chloride reference electrode using an IPC-Pro MF potentiostat (production of IPhChE RAS). Impedance spectra were studied in the frequency range $(\omega / 2 \pi)$ of $10 \mathrm{kHz}-0.05 \mathrm{~Hz}$ with an alternating voltage amplitude of $10 \mathrm{mV}$, using an electrochemical measuring complex from Solartron (UK) consisting of a 1255 SI impedance analyzer and a SI 1287 potentiostat [5]. Processing of the obtained results was realized according to the program ZView 3.0 allowing to make calculations for any equivalent circuits with a number of elements up to 20 .

\section{Results and discussion}

According to the gravimetric study, the INCORGAZ-111 inhibitor in NACE and M1 media with the addition of hydrogen sulfide exhibits a relatively high protective effect, increasing with concentration increase and reaching $80-90 \%$ at a concentration $200 \mathrm{mg} / \mathrm{L}$ (Tables 1 and 2).

An analysis of the polarization curves in investigated solutions indicates that the INCORGAZ-111 inhibitor in both media in the presence of hydrogen sulfide slows down the anodic process with a slight acceleration of the cathode one (Figure 1).

The protective effectiveness of the inhibitor studied in simulated stratum water is confirmed by the impedance measurement results. The hodographs obtained are a combination of several semicircles, whose diameters increase with the increasing exposure time of the electrode in solution. 
Table 1. Corrosion rate $\left(K, \mathrm{~g} /\left(\mathrm{m}^{2} \cdot \mathrm{h}\right)\right.$, numerator) and value of protective effect $(Z, \%$ denominator $)$ in the NACE medium in the presence of hydrogen sulfide and INCORGAZ-111 inhibitor.

\begin{tabular}{ccccc}
\hline \multirow{2}{*}{$\boldsymbol{C}_{\text {inh, } \mathbf{~ m g / L ~}}$} & \multicolumn{4}{c}{$\boldsymbol{C}_{\mathbf{H}_{\mathbf{2}} \mathbf{S}, \mathbf{m g} / \mathbf{L}}$} \\
\cline { 2 - 5 } & 50 & 100 & 200 & 400 \\
\hline 25 & $0.1277 / 48$ & $0.1101 / 47$ & $0.2117 / 42$ & $0.1981 / 74$ \\
50 & $0.0942 / 62$ & $0.0926 / 55$ & $0.1075 / 71$ & $0.1136 / 85$ \\
100 & $0.0744 / 70$ & $0.0878 / 58$ & $0.1216 / 67$ & $0.2273 / 70$ \\
200 & $0.0626 / 75$ & $0.0393 / 81$ & $0.0629 / 81$ & $0.0767 / 90$ \\
\hline
\end{tabular}

Table 2. Corrosion rate $\left(K, \mathrm{~g} /\left(\mathrm{m}^{2} \cdot \mathrm{h}\right)\right.$, numerator) and the value of the protective effect $(Z, \%$ denominator) in M1 medium in the presence of hydrogen sulfide and INCORGAZ-111 inhibitor.

\begin{tabular}{ccccc}
\hline \multirow{2}{*}{$\boldsymbol{C}_{\text {inh, } \mathbf{~ m g / L}}$} & \multicolumn{4}{c}{$\boldsymbol{C}_{\mathbf{H}_{\mathbf{2}} \mathbf{s}}, \mathbf{m g} / \mathbf{L}$} \\
\cline { 2 - 5 } & 50 & 100 & 200 & 400 \\
\hline 25 & $0.065 / 24$ & $0.027 / 69$ & $0.069 / 38$ & $0.308 / 27$ \\
50 & $0.037 / 56$ & $0.021 / 76$ & $0.043 / 62$ & $0.155 / 63$ \\
100 & $0.035 / 58$ & $0.016 / 82$ & $0.019 / 83$ & $0.081 / 81$ \\
200 & $0.027 / 68$ & $0.009 / 90$ & $0.012 / 90$ & $0.043 / 90$ \\
\hline
\end{tabular}

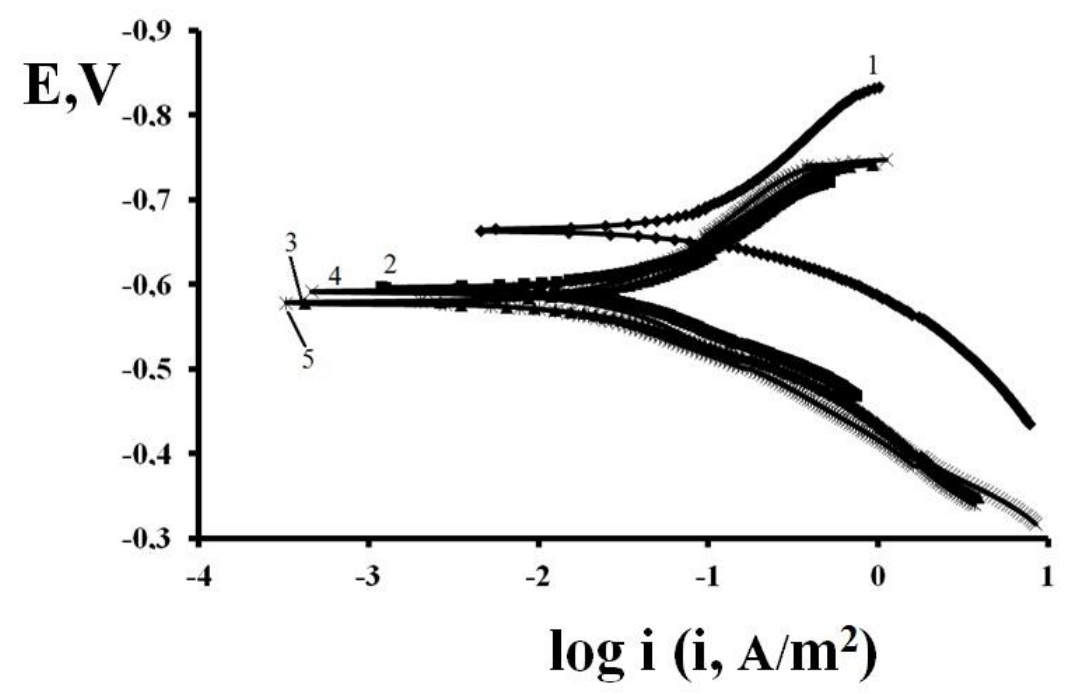

Figure 1. Polarization curves measured on steel in the $\mathrm{M} 1$ medium with $100 \mathrm{mg} / \mathrm{L} \mathrm{H}_{2} \mathrm{~S}$ and an INCORGAZ-111 inhibitor, mg/L: $1-0 ; 2-25 ; 3-50 ; 4-100 ; 5-200$.

This indicates an increase in the total resistance of the system and a decrease in the corrosion rate of steel due to formation of a sulfide film on the surface and adsorption of the inhibitor (Figure 2). 
These hodographs experience a transition from an arc to a linear shape what is probably conditioned by surface film porosity.

To process the experimental data of the electrochemical impedance, the equivalent electrical circuit shown in Figure 3 has been considered. In it, $R_{\mathrm{S}}$ is the resistance of the solution, $R_{1}$ and $R_{2}$ are the charge transfer resistance of the anodic and cathodic partial electrode reactions, respectively, $C_{\mathrm{dl}}$ - is the double layer capacitance, $C_{\mathrm{a}}$ and $R_{\mathrm{a}}$ are the capacitance and transfer resistance of the intermediate adsorbed particles formed during the anodic ionization of steel in hydrogen sulfide media, $Z(D)$ is Warburg diffusion impedance.

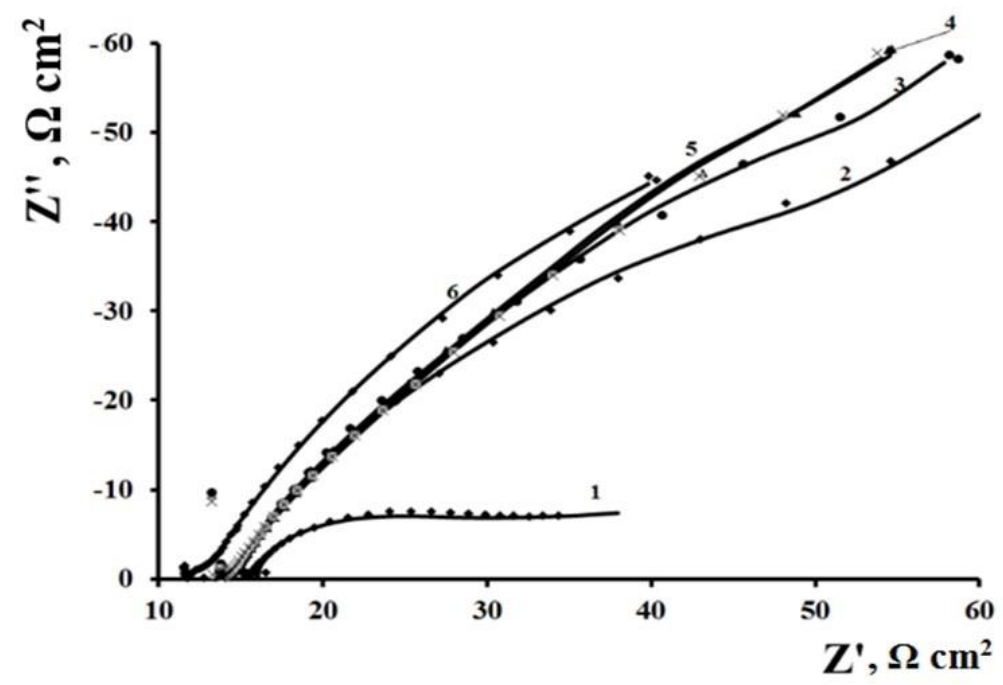

Figure 2. Nyquist diagram for $E_{\text {cor }}$ steel electrode in NACE medium containing $400 \mathrm{mg} / \mathrm{L}$ $\mathrm{H}_{2} \mathrm{~S}$ and $25 \mathrm{mg} / \mathrm{L}$ INCORGAZ-111 inhibitor. The exposure time of the electrode in solution, $h$ : $1-0.25 ; 2-0.5 ; 3$ to $1 ; 4$ to $2 ; 5$ to $4 ; 6-6$. (The points correspond to the experimental data, the solid line corresponds to the data calculated on the basis of the equivalent circuit).

Table 3 shows the data obtained after 6 hours in NACE medium with $400 \mathrm{mg} / \mathrm{L}$ of hydrogen sulfide and all tested inhibitor concentrations. From Table 3 it follows that with increasing concentration of the inhibitor INCORGAZ-111 the value of resistance of charge transfer in the anodic reaction $R_{1}$ increases with a slight change in the resistance of charge transfer in the cathodic reaction $R_{2}$.

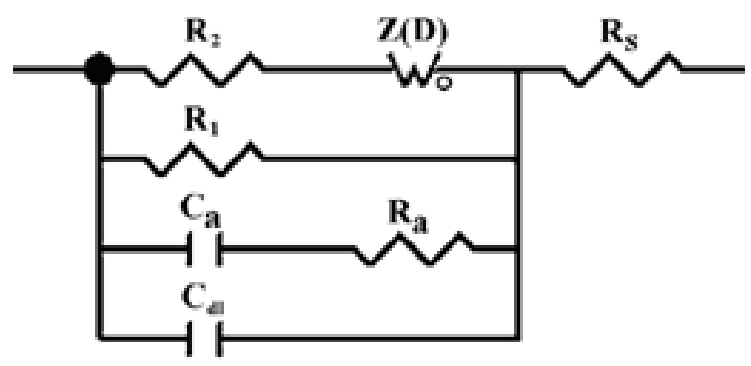

Figure 3. An equivalent circuit simulating the behavior of carbon steel with a corrosion potential in the studied solutions saturated with hydrogen sulfide. 
This is consistent with the results of polarization measurements, indicating an inhibition of the anodic process with increase of the inhibitor concentration. The diffusion resistance of the cathode depolarizer $R_{\mathrm{D}}$ is either comparable with the value of $R_{1}$, or exceeds it by $2-$ 4 times at different concentrations of the inhibitor, but this does not necessarily indicate that mass transfer limits the corrosion process what is possible only when the process is proceeding at the limiting cathode current. And since the cathodic reaction does not determine the corrosion process, even at $R_{\mathrm{D}}>R_{1}$ the process is limited by the anodic reaction kinetics.

Table 3. Numerical values of elements of the equivalent circuit at $E_{\text {cor }}$ of the steel electrode in the NACE medium containing $400 \mathrm{mg} / \mathrm{L} \mathrm{H}_{2} \mathrm{~S}$ and the INCORGAZ-111 inhibitor after 6 hours of exposure of the electrode in solution.

\begin{tabular}{cccccc}
\hline \multirow{2}{*}{ Parameter } & \multicolumn{5}{c}{ Cinh, $\mathbf{m g} / \mathbf{L}$} \\
\cline { 2 - 6 } & 0 & 25 & 50 & 100 & 200 \\
\hline$R_{2}, \Omega \cdot \mathrm{cm}^{2}$ & 13.52 & 14.81 & 14.20 & 10.27 & 3.85 \\
$R_{\mathrm{D}}, \Omega \cdot \mathrm{cm}^{2}$ & 947.7 & 467.6 & 895.0 & 331.3 & 567.2 \\
$\tau, \mathrm{s}$ & 20.34 & 60.19 & 89.10 & 30.24 & 51.08 \\
$p$ & 0,68 & 0.72 & 0.63 & 0.61 & 0.67 \\
$R_{1}, \Omega \cdot \mathrm{cm}^{2}$ & 374.5 & 394.8 & 406.8 & 783 & 2623 \\
$C_{\mathrm{a}}, \mathrm{F} / \mathrm{cm}^{2}$ & $1.7 \cdot 10^{-3}$ & $6.9 \cdot 10^{-3}$ & $6.5 \cdot 10^{-3}$ & $1.2 \cdot 10^{-3}$ & $5.4 \cdot 10^{-4}$ \\
$R_{\mathrm{a}}, \Omega \cdot \mathrm{cm}^{2}$ & 12.53 & 10.8 & 11.31 & 51.56 & 9.167 \\
$C_{\mathrm{d} \mathrm{l}}, \mu \mathrm{F} / \mathrm{cm}^{2}$ & 5.06 & 4.89 & 2.62 & 1.67 & 1.28 \\
$R_{\mathrm{s}}, \Omega \cdot \mathrm{cm}^{2}$ & 13.4 & 14.0 & 12.39 & 12.0 & 18.0 \\
Error & $0.019 / 1.89$ & $0.017 / 1.75$ & $0.013 / 1.85$ & $0.003 / 0,31$ & $0.003 / 0.31$ \\
\hline
\end{tabular}

As the inhibitor concentration increases, a decrease in the capacitance of the electric double layer $C_{\mathrm{dl}}$ takes place. This indicates an adsorption of the inhibitor on the electrode surface. The results obtained allow to calculate electrode surface coverage $\Theta$ with the inhibitor according to the formula [6]

$$
\Theta=\left(C_{0}-C\right) /\left(C_{0}-C_{1}\right),
$$

where $C_{0}, C$ and $C_{1}$ are the capacities of the electric double layer in the solution without addition of an inhibitor, with that and with the maximum coverage of the electrode surface with particles of the inhibitor, respectively.

The calculation of $C_{1}$ is performed according to the dependence $C_{\mathrm{dl}}=C_{\mathrm{dl}}\left(1 / C_{\mathrm{inh}}\right)$ (Figure 4). 


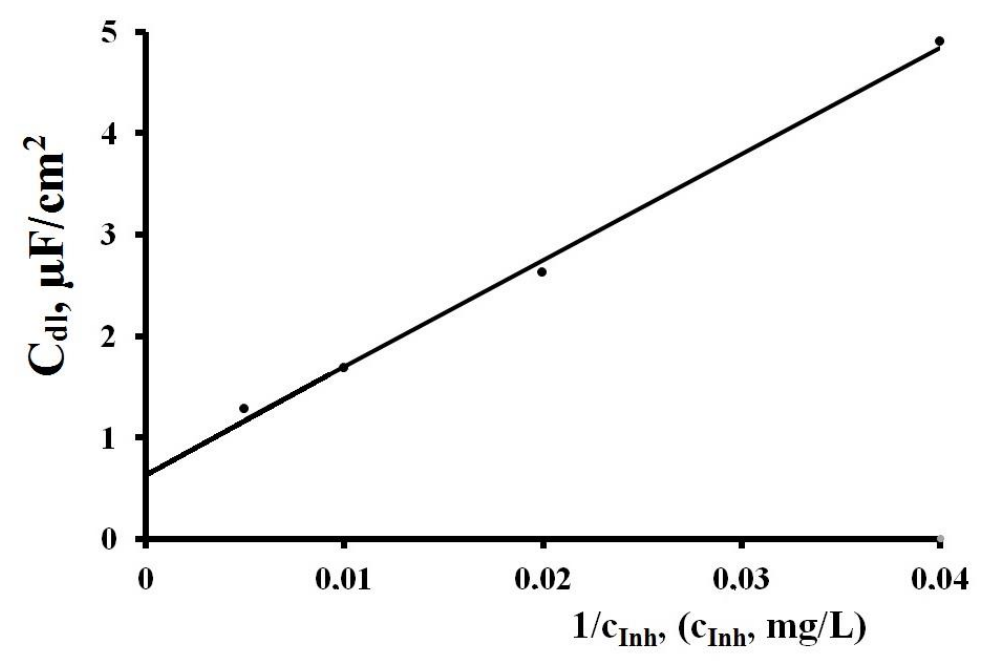

Figure 4. The dependence of $C_{\mathrm{dl}}, \mu \mathrm{F} / \mathrm{cm}^{2}$, on $1 / C_{\mathrm{inh}}$ in NACE medium containing $400 \mathrm{mg} / \mathrm{L}$ $\mathrm{H}_{2} \mathrm{~S}$, after 6 hours of exposure.

The value of $C_{1}$ calculated according to Figure 4 is equal to $0.7 \mu \mathrm{F} / \mathrm{cm}^{2}$. The corresponding coverage $\Theta$ of the electrode surface with an INCORGAZ-111 inhibitor at different concentrations is presented in Table 4.

From Table 4 it follows that after 6 hours of exposure of the electrode in solution, a significant adsorption of the inhibitor on the surface takes place.

Numerical values of the elements of the equivalent circuit after 24 hours of exposure of the steel electrode in the M1 medium containing $50 \mathrm{mg} / \mathrm{L} \mathrm{H}_{2} \mathrm{~S}$ and all the tested concentrations of the INCORGAZ-111 inhibitor are presented in Table 5.

Table 4. The values of the electrode surface coverage with INCORGAZ-111 inhibitor in NACE medium with $400 \mathrm{mg} / \mathrm{L}$ of hydrogen sulfide.

\begin{tabular}{ccccc}
\hline $\boldsymbol{C}_{\text {inh, }}, \mathbf{m g} / \mathbf{L}$ & 25 & 50 & 100 & 200 \\
\hline $\boldsymbol{\Theta}$ & 0.04 & 0.56 & 0.78 & 0.87 \\
\hline
\end{tabular}

As in the previous case, with an increase in concentration of the INCORGAZ-111 inhibitor, the charge transfer resistance $R_{1}$ in the anodic reaction increases with a slight change in $R_{2}$, what is consistent with the polarization measurements.

The capacitance of the electric double layer naturally decreases at the same time, indicating the adsorption of the inhibitor.

The $C_{1}$ value necessary for calculating $\Theta$ by formula (1) is determined graphically on the basis of the linear dependence $C_{\mathrm{dl}}=C_{\mathrm{dl}}\left(1 / C_{\mathrm{inh}}\right)$ obtained according to the data in Table 5 . $C_{1}$ is equal to $2 \mu \mathrm{F} / \mathrm{cm}^{2}$. Coverage of the electrode surface with an INCORGAZ-111 inhibitor $(\Theta)$ obtained taking into account the value of $C_{1}$ at different concentrations is shown in Table 6. 
Table 5. Numerical values of the equivalent circuit elements at $E_{\text {cor }}$ of steel electrode in the M1 medium containing $50 \mathrm{mg} / \mathrm{L} \mathrm{H}_{2} \mathrm{~S}$ and the INCORGAZ-111 inhibitor after 24 hours of exposure of the electrode in solution.

\begin{tabular}{cccccc}
\hline \multirow{2}{*}{ Parameter } & \multicolumn{5}{c}{$\boldsymbol{C}_{\mathbf{i n h}, \mathbf{~ m g} / \mathbf{L}}$} \\
\cline { 2 - 6 } & 0 & 25 & 50 & 100 & 200 \\
\hline$R_{2}, \Omega \cdot \mathrm{cm}^{2}$ & 2.34 & 0.56 & 2.69 & 3.41 & 5.76 \\
$R_{\mathrm{D}}, \Omega \cdot \mathrm{cm}^{2}$ & 619.1 & 796.9 & 811.6 & 1057 & 1141 \\
$\tau, \mathrm{s}$ & 8.18 & 34.02 & 4.48 & 1.72 & 2.22 \\
$p$ & 0.71 & 0.50 & 0.49 & 0.55 & 0.49 \\
$R_{1}, \Omega \cdot \mathrm{cm}^{2}$ & 56.29 & 126.7 & 271.4 & 321.7 & 594.2 \\
$C_{\mathrm{a}}, \mathrm{F} / \mathrm{cm}^{2}$ & $4.2 \cdot 10^{-3}$ & $9.0 \cdot 10^{-5}$ & $8.0 \cdot 10^{-3}$ & $1.5 \cdot 10^{-3}$ & $2.3 \cdot 10^{-3}$ \\
$R_{\mathrm{a}}, \Omega \cdot \mathrm{cm}^{2}$ & 0.3942 & 36.54 & 366.3 & 452.5 & 596.9 \\
$C_{\mathrm{d} \mathrm{d}, \mu \mathrm{F} / \mathrm{cm}^{2}}$ & 48.0 & 39.4 & 15.4 & 9.4 & 6.9 \\
$R_{\mathrm{s}}, \Omega \cdot \mathrm{cm}^{2}$ & 5.747 & 12.1 & 10.62 & 11.62 & 12.62 \\
Error & $2.7 \cdot 10^{-3} / 0.27$ & $7.6 \cdot 10^{-4} / 0.08$ & $4.7 \cdot 10^{-4} / 0.05$ & $1.1 \cdot 10^{-3} / 0.11$ & $6.8 \cdot 10^{-4} / 0.07$ \\
\hline
\end{tabular}

Table 6. The values of the electrode surface coverage with INCORGAZ-111 inhibitor in M1 medium with $50 \mathrm{mg} / \mathrm{L}$ of hydrogen sulfide.

\begin{tabular}{ccccc}
\hline $\boldsymbol{C}_{\text {inh, }}, \mathbf{m g} / \mathrm{L}$ & 25 & 50 & 100 & 200 \\
\hline $\boldsymbol{\Theta}$ & 0.19 & 0.71 & 0.84 & 0.89 \\
\hline
\end{tabular}

An attempt to approximate the $\Theta\left(\log C_{\text {inh }}\right)$ dependence for the NACE medium with $400 \mathrm{mg} / \mathrm{L}$ hydrogen sulfide and the $\mathrm{M} 1$ medium with $50 \mathrm{mg} / \mathrm{L} \mathrm{H}_{2} \mathrm{~S}$ in the presence of INCORGAZ-111 inhibitor does not seem to be correct with a straight line (Figure 5), experimental points in this coordinate system form a convex curve. With obviousness, the adsorption of the inhibitor on the surface of the electrode is described by a different isotherm than the Temkin isotherm, since there is no pronounced linearized region.

To interpret the results of Figure 5 we use the polylogarithmic isotherm [7], generalizing the Temkin isotherm to the case of linear energy heterogeneity of the surface.

Since this isotherm has been recently derived and not received wide publicity yet, we provide some basic information about it.

If the probability to meet an adsorption center with a heat of adsorption within the segment $[\varepsilon, \varepsilon+\delta \varepsilon]$ is a linear function of $\varepsilon$ rather than a constant, as in the case of the Temkin isotherm, the polylogarithmic adsorption isotherm arises then:

$$
\Theta(x)=\gamma\left[\operatorname{Li}_{2}\left(-a_{+} x\right)-\operatorname{Li}_{2}\left(-a_{-} x\right)\right]+\frac{1}{f} \log \frac{1+a_{+} x}{1+a_{-} x}+\gamma \frac{f}{2} \log \left[\left(1+a_{+} x\right)\left(1+a_{-} x\right)\right],
$$


where $f$ is Temkin's usual energy heterogeneity parameter referred to as energy dispersion in the case of a polylogarithmic isotherm, $x$ is volume concentration of adsorbate particles, coefficients

$$
a_{+}=a_{0} \exp \left(\frac{f}{2}\right) \exp \left(\frac{\varepsilon_{0}}{k T}\right) \text { and } a_{-}=a_{0} \exp \left(-\frac{f}{2}\right) \exp \left(\frac{\varepsilon_{0}}{k T}\right)
$$

correspond to strongly and weakly adsorbing surface areas, respectively, $\varepsilon_{0}$ is the middle of the entire range of adsorption heats and $\mathrm{Li}_{2}$ is the polylogarithmic function of the second order [8] whose the isotherm has adopted.
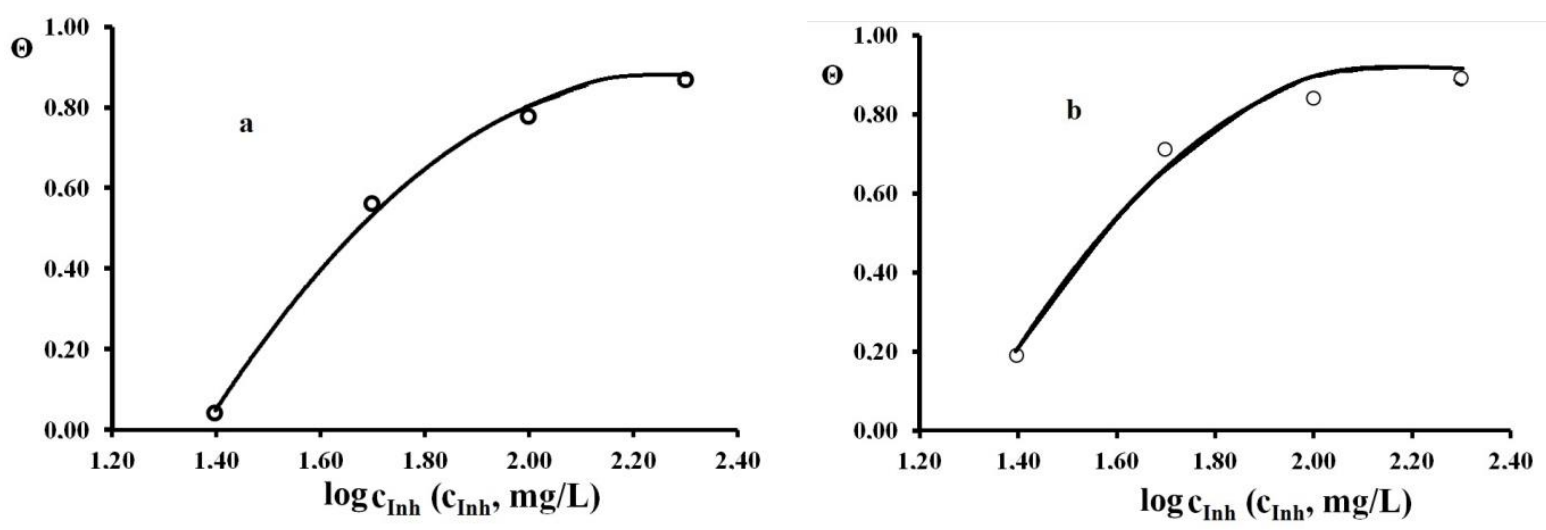

Figure 5. Dependence $\Theta\left(\log C_{\text {inh }}\right)$ for the NACE medium with $400 \mathrm{mg} / \mathrm{L} \mathrm{H}_{2} \mathrm{~S}$ (a) and the M1 medium with $50 \mathrm{mg} / \mathrm{L} \mathrm{H}_{2} \mathrm{~S}$ (b) and with an INCORGAZ-111 inhibitor.

Whether the linear distribution function of adsorption centers on adsorption heat $\epsilon$ is increasing or decreasing depends on the sign of energy gradient $\gamma$ : Similar to the Temkin isotherm, the polylogarithmic isotherm (2) can be simplified in the region of "average" coverages [7], taking the form

$$
\Theta(x)=\frac{1}{f} \log \left(a_{+} x\right)+\gamma\left[-\frac{\pi^{2}}{6}-\frac{1}{2} \log ^{2}\left(a_{+} x\right)+\frac{f}{2} \log \left(a_{+} x\right)\right]
$$

Let us consider the limiting case of a large energy gradient, when the distribution function of the adsorption centers strongly depends on the heat of adsorption. $|\gamma|=2 / f^{2}$ takes place then. The case $\gamma=-2 / f^{2}$ characteristic for surfaces named in [7] as moderate and having the greater number of adsorption centers, the lower their heat of adsorption is with respect to this adsorbate, does not have anything common with the data in Figure 5, since it forms a concave curve in this coordinate system (a quasi-parabola).

The case $\gamma=2 / f^{2}$, which characterizes adsorption-overactive surfaces for which a larger number of adsorption centers corresponds to a higher heat of adsorption, allows satisfactory approximation of the experimental data. Upon substituting this value of the energy gradient into equation (3), we obtain 


$$
\Theta(x)=-\left[\frac{1}{f} \log \left(a_{+} x\right)-1\right]^{2}+1-\frac{\pi^{2}}{3 f^{2}}
$$

The dependence $\Theta=\Theta(x)$ does not enable us to obtain an analytical solution in the leastsquares method to find the energy gradient and the constant of adsorption equilibrium because of rather cumbersome equations. However, the transition to the inverse function using the logarithm of concentration $x \equiv C_{\text {inh }}$ :

$$
\log C_{\text {inh }}=-\log a_{+}+f\left[1-\sqrt{1-\frac{\pi^{2}}{3 f^{2}}-\Theta}\right]
$$

enables one to obtain substantially simpler equations, one of which is an explicit dependence of the adsorption equilibrium constant $a_{+}$on energy dispersion $f$ and another equation, for energy dispersion, is easy to solve numerically what gives values $\ln a_{+}=-1.50$ and $f=5.32$ for the data in Table 4 (Figure 5a). Figure 6 demonstrates a better approximation by the polylogarithmic isotherm in the entire concentration range compared to the Frumkin isotherm, which gives a very significant deviation at low concentrations and, in general, experiences an inflection in these coordinates, free of which are both the polylogarithmic isotherm and the experimental data. The approximation result by the polylogarithmic isotherm can be further improved with a smaller value of the energy gradient (the radius of curvature will decrease in this case).

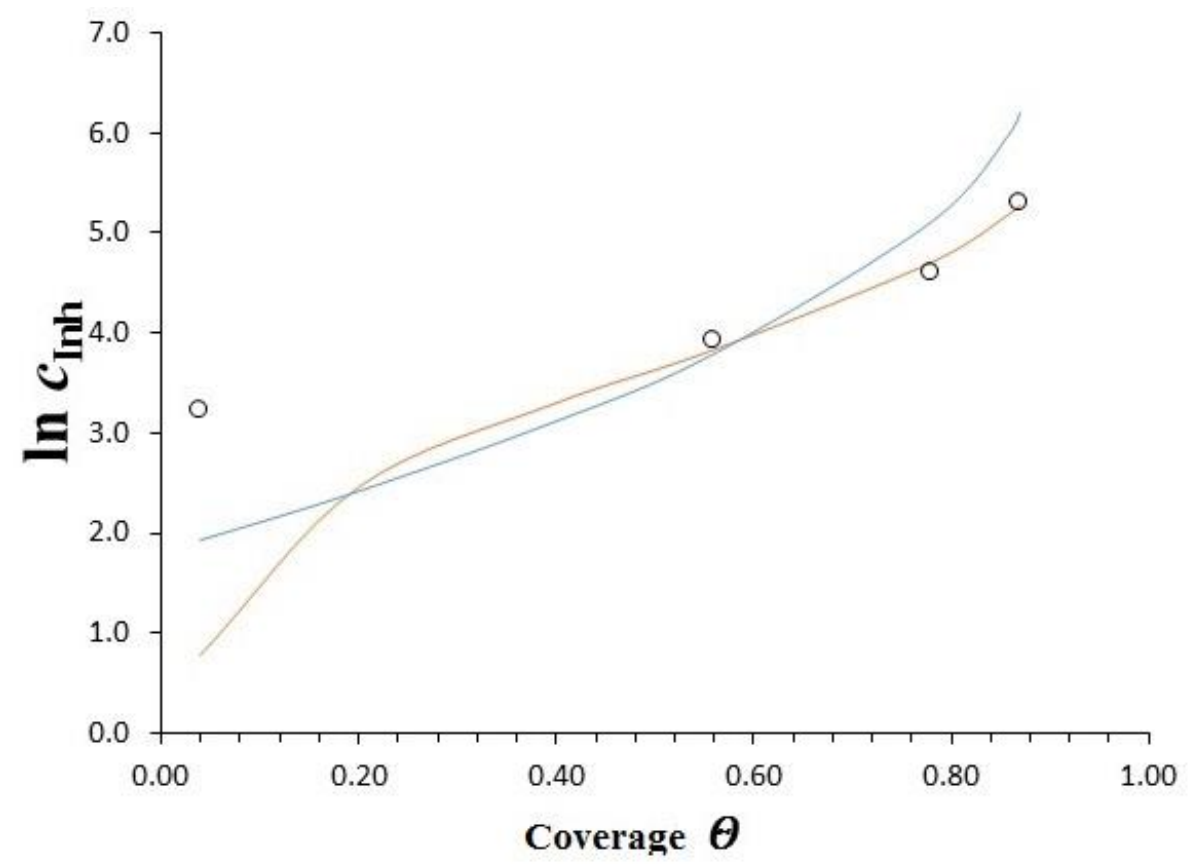

Figure 6. Approximation of the measurement data (Table 4) by the polylogarithmic isotherm (blue curve) and the Frumkin isotherm (yellow). 
Thus, the experimental data in Table 4 evidence in favor of a linear energy heterogeneity of the surface with the energy dispersion of $f=5.32$ and the energy gradient close to $\gamma=\frac{2}{f^{2}} \approx 0.071$.

The nature of the dependence $\Theta\left(\log C_{\mathrm{inh}}\right)$ for the $\mathrm{M} 1$ medium with $50 \mathrm{mg} / \mathrm{L}$ hydrogen sulfide in the presence of an INCORGAZ-111 inhibitor (Figure 5b) makes it reasonable to perform the analysis in full analogy with the above said for Figure 5a what leads to the following values of the adsorption parameters on an energetically linearly heterogeneous surface: adsorption constant $\ln a_{+}=-0.86$, energy dispersion $f=5.53$ and energy gradient close to $\gamma=\frac{2}{f^{2}} \approx 0.066$.

The value of the adsorption equilibrium constant $a_{+}$enables us to calculate the value of free adsorption energy $\Delta G_{\text {ads }}^{0}$ according to the equation

$$
\Delta G_{\mathrm{ads}}^{0}=-R T \ln \left(a_{+} \cdot 10^{6}\right)
$$

where $10^{6}$ is the concentration of water in water, $\mathrm{mg} / \mathrm{L}$.

The value of $-\Delta G_{\text {ads }}^{0}$ for the INCORGAZ-111 inhibitor at a temperature of $298 \mathrm{~K}$ in NACE medium with $400 \mathrm{mg} / \mathrm{L} \mathrm{H}_{2} \mathrm{~S}$ and M1 with $50 \mathrm{mg} / \mathrm{L} \mathrm{H}_{2} \mathrm{~S}$ is characterized by the values of 30.5 and $32.1 \mathrm{~kJ} / \mathrm{mol}$ respectively, indicating the physical nature of adsorption of the inhibitor. In addition, there is practically no influence of the medium on $\Delta G_{\text {ads }}^{0}$.

\section{Conclusions}

1. The protective efficacy of the inhibitory composition INCORGAZ-111 against hydrogensulfide corrosion of carbon steel in the model stratum waters is shown by means of the gravimetry and electrochemical polarization methods and by impedance measurements.

2. The inhibitor adsorption was confirmed by the electrochemical impedance spectroscopy method. The patterns of adsorption in the entire measurement range are satisfactorily described by the polylogarithmic isotherm with a large energy gradient of the adsorption centers, what testifies to the linear energy inhomogeneity of the surface.

3 . The calculated values of free energy of adsorption indicate the physical character of adsorption of the inhibitor.

\section{References}

1. N.V. Shel and K.O. Strelnikova, Some methodological aspects of studying the inhibition of hydrogen sulfide corrosion of steel, Korroz.: mater., zashch. (Corrosion: materials, protection), 2012, no. 4, 13-19 (in Russian).

2. N.P. Andreeva, A.V. Larionov, O.Yu. Grafov, A.S. Semeikin, L.P. Kazanskii and Yu.I. Kuznetsov, Adsorption of 5-(4'-aminophenyl)-10,15,20-(4'-sulfophenyl)porphin 
on silver surface, Korroz.: mater., zashch. (Corrosion: materials, protection), 2019, no. 3, 16-22 (in Russian). doi: 10.31044/1813-7016-2019-0-3-16-22

3. Yu.I. Kuznetsov, M.O. Agafonkina, N.P. Andreeva and D.B. Vershok, Adsorption of 2mercaptobenzthiazol on copper and MNZh-5-1 alloy and their protection from corrosion in aqueous solutions, Int. J. Corros. Scale Inhib., 2020, 9, no. 1, 344-361. doi: 10.17675/2305-6894-2020-9-1-23

4. N.P. Andreeva, M.O. Agafonkina and Yu.I. Kuznetsov, Adsorption of 1,2,3benzotriazole on copper from a borate buffer solution, Korroz.: mater., zashch. (Corrosion: materials, protection), 2010, no. 9, 7-11 (in Russian).

5. L.E. Tsygankova, M.N. Esina, V.I. Vigdorovich and N.V. Shel, Study of steel corrosion inhibition in media containing $\mathrm{H}_{2} \mathrm{~S}$ and $\mathrm{CO}_{2}$ by impedance spectroscopy and polarization resistance methods, Int. J. Corros. Scale Inhib., 2014, 3, no. 1, 48-58. doi: 10.17675/2305-6894-2014-3-1-048-058

6. B.B. Damaskin, O.A. Petriy and V.V. Batrakov, Adsorption of organic compounds on electrodes, Moscow, Nauka, 1968, 334 (in Russian).

7. M. Vigdorowitsch, L.E. Tsygankova and V.D. Prokhorenkov, The polylogarithmic adsorption function: a linear energetic surface heterogeneity, J. Math. Chem., 2020, 58, 1353-1363. doi: 10.1007/s10910-020-01133-2

8. L.C. Maximon, The dilogarithm function for complex argument, Proc. R. Soc. London, Ser. A, 2003, 459, 2807-2819. doi: $10.1098 /$ rspa.2003.1156 